\title{
Nivel de capacitación del capital humano y la calidad de servicio en los empresarios de las PYMES del centro comercial Plaza Josfel, en la localidad de Ate Vitarte, en el periodo 2016
}

Level of training of the human capital and the quality of service in the businessmen of the SMES of the mall Plaza Josfel, in the locality Ate Vitarte, in the period 2016

\author{
Yuliana Margot Ñahui Gutiérrez ${ }^{11}$; Kelly Maricet Villoslada Romero ${ }^{1}$ \\ ${ }^{1}$ Escuela Profesional de Administración, Facultad de Ciencias Empresariales, Universidad Peruana Unión
}

\section{INFORMACIÓN DEL ARTÍCULO}

Historia del artículo

Recibido: 21 de junio 2016

Aceptado: 06 de octubre del 2016

Palabras clave:

Capital humano, calidad de servicio, capacitación.
Keywords:

Human capital, quality of service, training.

\section{Resumen}

El propósito de la presente investigación buscó determinar la relación existente entre el nivel de capacitación del capital humano y la calidad de servicio percibida en los empresarios de las PYMES del centro comercial Plaza Josfel, en Ate Vitarte. La investigación está desarrollada bajo el diseño no experimental transaccional, con estudio descriptivo y correlacional, teniendo como variable independiente el nivel de capacitación del capital humano y como variable dependiente la calidad de servicio. Participaron 306 empresarios, muestra calculada de una población de 1500. Según los resultados se determinó que existe relación positiva (169.602) significativa ( $p$ valor 0.000 ) entre el nivel de capacitación del capital humano y calidad de servicio. De igual modo existe relación positiva (297.967) significativa ( $p$ valor 0.000 ) entre el nivel de capacitación del capital humano y la atención a tiempo de pedido. También existe relación positiva (134.655) significativa ( $p$ valor 0.000 ) entre el nivel de capacitación del capital humano y el buen trato al cliente. Por último, existe relación (238.980) significativa ( $p$ valor 0.000) entre el nivel de capacitación del capital humano y la entrega oportuna de pedido. En conclusión, el nivel de capacitación del capital humano se relaciona favorablemente con la calidad de servicio, así como con la atención a tiempo de pedido, buen trato al cliente, entrega oportuna del pedido, en los empresarios de las PYMES del centro comercial Plaza Josfel.

\section{Abstrac}

The intention of the present investigation sought to determine the existing relation between the level of training of the human capital and the quality of service perceived in the businessmen of the SMES of the Mall Plaza Josfel, in Tie Vitarte. The investigation is developed under the not experimental transactional design, with descriptive study and correlacional, taking as an independent variable the level of training of the human capital and as variable dependent the quality of service. There took part 306 businessmen, sample calculated of a population of 1500. According to the results one determined that there exists positive relation (169.602) significant ( $p$ value 0.000) between the level of training of the human capital and quality of ser-

\footnotetext{
Autor correpondiente: Yuliana Margot Ñahui Gutiérrez Tel.: +51-977-765-649

Correo electrónico: yulianamargot@gmail.com
} 
vice. Of equal way there exists positive relation (297.967) significant ( $p$ value 0.000 ) between the level of training of the human capital and the attention in time of order. Also there exists positive relation (134.655) significant ( $p$ value 0.000 ) between the level of training of the human capital and the good treatment to the client. Finally, there exists relation (238.980) significant ( $p$ value 0.000 ) between the level of training of the human capital and the opportune delivery of order. In conclusion, the level of training of the human capital relates favorably to the quality of service, as well as to the attention in time of order, good treatment to the client, opportune delivery of the order in the businessmen of the SMES of the mall Plaza Josfel.

\section{Introducción}

El mundo enfrenta cambios sin precedentes. Vemos como las nuevas tecnologías impulsan el desarrollo de dinámicas económicas, culturales, y sociales que plantean nuevos desafíos a las formas de producción. Generar un diálogo entre el mundo de la formación y el mundo del trabajo es un movimiento estratégico para asegurar el cumplimiento mutuo de expectativas entre trabajo y formación, y para formar capital humano de calidad (Rivera, 2005).

Nonaka y Takeuri (1992), citado por Madrigal (2009), señala que el capital humano es crear nuevos conocimientos, significa recrear la empresa y a cada uno de los que la conforman un interrumpido proceso de renovación organizacional y personal; dirigido a alcanzar habilidades, destrezas y valores en los trabajadores.

En cuanto a calidad, Carlos, Montelongo y Nájera (2010) mencionan que esta se produce durante la prestación del servicio, generalmente en una interacción entre el cliente y el personal de contacto de la organización, por lo que la calidad del servicio se conceptúa como la discrepancia entre las expectativas del cliente y sus percepciones del servicio recibido y depende en gran medida del desempeño de los empleados. Mientras que para Dávila, Coronado, y Cerecer (2012), la calidad de servicio es un concepto complicado de abordar debido a la característica de intangibilidad que compone al servicio, los problemas en la recepción, la producción en tiempo real y la diferenciación entre la calidad humanística y mecanicista.

García y Edel (2008) expresan que un factor principal de una empresa, en el nivel de competitividad y productividad, es su gente. La empresa puede contar con la mejor infraestructura, los mejores sistemas y todos los recursos para operar, pero si no invierte en el factor más importante, que es el capital humano, es difícil que llegue a los objetivos planeados. En consecuencia, el capital humano de una empresa, organización, región o país es la piedra angular, es la fuente de desarrollo y uno de los factores que generan la competitividad (Madrigal, 2009).

Cotte y Cotrino (2006) señalan que los conocimientos y habilidades de la población trabajadora inciden positivamente en su productividad. En este desarrollo se adoptan nuevas tecnologías, en cuyos contextos se distinguen dos efectos del capital humano sobre el crecimiento económico: para mayor productividad o eficiencia se adquiere mayores niveles de educación o entrenamiento; y el beneficio generado por mayores niveles de preparación; es decir, es un efecto social no individual, indicando que economías con mayores niveles o mayores promedios de capital humano capacitado son más productivas que las demás. 
Según estudios de ManpowerGroup (2013) a nivel mundial, el 35\% de casi 38.000 empleadores encuestados están teniendo dificultades para cubrir puestos debido a la falta de talento disponible, y esto impacta en el servicio al cliente. Un porcentaje de empleadores reportan que la escasez de talento marca sus resultados de negocio en un nivel alto, que subió del 13\% en 2012 a 19\% en 2013. La escasez de talento más aguda fue reportada por empleadores de Japón con 85\%, Brasil con $68 \%$, India con 61\%, Turquía 58\%, Hong Kong 57\%, Bulgaria (54\%) y Rumania (54\%).

Sin embargo, Alvarado Daga (2013) destaca que en el Perú la gestión de la calidad es reconocida a través del otorgamiento del Premio Nacional a la Calidad, denominado CDI 2013 (centro de desarrollo industrial), a las empresas que han demostrado ser muy eficientes debido a la gestión del talento humano mediante la capacitación continua, instrumento diseñado para orientar a las organizaciones en sus procesos de mejora permanente e incrementar sus condiciones de competitividad.

Por todas estas razones expuestas, es necesario realizar este estudio, a fin de conocer nivel de capacitación del capital humano y su relación con calidad de servicio en las PYMES del centro comercial Plaza Josfel en Ate Vitarte.

\section{Revisión de la literatura}

\section{Capital humano}

Según Nonaka y Takeuri, citado por Madrigal (2009), el capital humano propende a la creación de nuevos conocimientos, en un proceso de renovación organizacional y personal. Madrigal (2009) menciona que el conocimiento se adquiere a lo largo de su vida, producien- do bienes, servicios e ideas bajo condicionantes del mercado. Tal conocimiento, primero, es tácito o explicito, poseído por las personas y equipos; y segundo, el capital humano se complementa con la capacidad de regenerar el conocimiento; es decir, con la capacidad de aprender (Selva, 2004).

Por otro lado, Valencia (2005) menciona que el capital humano constituye un activo intangible como la capacidad que incrementa la productividad, impulsa la innovación y con ella, la competitividad. Es un recurso valioso, cuya gestión no solo implica una adecuada compensación económica por su labor, sino también participe de los retos de la empresa, de modo que se sienta parte de ella y que adopte una actitud de compromiso con todos los grupos de interés de la organización (Santos, 2012). Asimismo, Ospina, Stella, y Estrada (2004) afirman que los activos más valiosos de las empresas, son los activos intangibles, basados en conocimientos, habilidades, valores y actitudes de las personas que forman su núcleo estable.

Teorías del capital humano

El devenir de las nociones del capital humano es desarrollado por los autores: Schultz, Becker y Blaug.

Schultz, identifica la dificultad para medir los beneficios de la inversión en capital humano, el cual señala que el problema se origina en cómo uno puede diferenciar los gastos de consumo de los gastos de inversión; para ello la solución de esta cuestión propone un método alternativo: calcular la inversión humana a través de su rendimiento más que a través de su costo (Villalobos y Pedroza, 2009). El costo debe ser evaluado por las ventajas que genera al mejorar la competencia y productividad a partir de los métodos 
utilizados en los análisis costo beneficio y costo eficiencia como efectos sobre la sociedad (Destinobles, 2006).

Segundo, Becker, citado por Villalobos y Pedroza (2009), calificó a la segunda mitad del siglo XX como la era de la gente, debido a que el desarrollo de una nación se mide por la utilización de los conocimientos, de las técnicas, de los hábitos de la población. Por ello a fines del siglo XXI, se establecieron en diversas compañías, los departamentos de bienestar, lo que ahora se conoce como áreas de capital humano (Werther, Davis y Guzmán, 2014).

Por último, Blaug, citado por Villalobos y Pedroza (2009), señala que el capital humano se caracterizaba por un individualismo metodológico, el cual no consideraba la formación del capital humano. García (1991) menciona que la educación en la formación del capital humano es considerada como una inversión que contribuye al aumento del capital humano, tanto para el demandante de educación como para la sociedad en su conjunto. De tal manera la educación, como inversión, implica el empleo del capital para obtener un beneficio en el futuro, dicha inversión se calcula de acuerdo al rendimiento, traduciéndose en que a mayor educación y menor edad, corresponderá mayor salario (Villalobos y Pedroza, 2009).

\section{Economía del conocimiento y capital humano}

La gestión del capital humano es generado por el conocimiento como recurso principal y el aprendizaje de la organización en el conocimiento.

Según, Madrigal (2009), la economía mundial se ha movido en su transformación de sociedad de la información (flujo de mensajes) a una del conocimiento, este último como el recurso principal del crecimiento, la competitividad, su empleo fortalece los recursos humanos y desarrollar el capital humano. Asimismo, se concibe la inversión en capital humano cuyos retornos podían ser capturados por los empleados con mayores salarios y por las empresas en mayor productividad (Mungaray y Ramírez, 2007).

El aprendizaje de la organización surge en la interacción de las personas en el proceso productivo, surge en el conocimiento del equipo de trabajo que responde ante problemas, motivando una mejor comunicación y organización que impacta al interior y en el mercado (Mungaray y Ramírez, 2007). Es decir, según Didier (2013), el capital humano refiere a los conocimientos y habilidades que poseen los individuos y que es adquirida a través de la educación y experiencia.

\section{Capital humano como ventaja competi-} tiva

Según Calderón y Naranjo (2004), la importancia de las personas en el desarrollo de las organizaciones construye ventaja competitiva fundamentada en el talento humano (capital humano) que responde a los nuevos retos. En este sentido la gestión de los recursos humanos tiene un carácter estratégico en el aumento de la competitividad de las empresas que involucre la introducción de nuevas tecnologías, el desarrollo del conocimiento organizacional y el servicio al cliente (Becerra y Álvarez, 2011).

Ramírez, citado por Lozano (2007), asume que el desarrollo del talento humano como ventaja competitiva requiere de tres aspectos básicos: capacidad, compromiso y acción. La capacidad es un conjunto de conocimientos, habilidades y competencias o actitudes (Lozano, 2007). Sin embargo, para Calderón y Na- 
ranjo (2004) mencionan que la capacidad permite a la persona controlar sus emociones en momentos críticos y aprovecharlos como retroalimentación para su crecimiento personal. El compromiso es el activo requerido para enfrentar las demandas de un entorno turbulento y alcanzar la misión organizacional (Madrigal, 2009). Para Lozano (2007), el compromiso es el motor para que el profesional aporte lo máximo posible y no se marche a otra compañía. La empresa que desarrolla las capacidades también puede fortalecer el compromiso mediante incentivos.

\section{Calidad del servicio}

La calidad de servicio puede ser entendido como la diferencia a partir de la percepción y como una filosofía empresarial. Según, Carlos, Montelongo y Nájera (2010), la calidad del servicio se conceptúa como la discrepancia entre las expectativas del cliente y sus percepciones del servicio recibido (Dávila, Coronado, y Cerecer, 2012). Aunque para Pinto, Férnandez, y Gómez (2007), la calidad de servicio se entiende desde el punto de vista de la percepción del usuario/cliente sobre el servicio, y no por las características intrínsecas del servicio ofrecido.

Por otro lado, Berry (2004), citado por Clemenza, Gotera, y Araujo (2010), plantea que la calidad de servicio es la filosofía basada en los principios del liderazgo con espíritu colectivo de servicio, proporcionando maneras eficaces para conservar a los clientes. Al mismo tiempo, involucra un servicio entregado por el personal administrativo con las instalaciones e infraestructura de la organización Torres y Araya (2010). Además, Lara (2002), dice que la calidad del servicio se puede mejorar mediante el compromiso total de la organización, siendo este compromiso como parte de la filosofía en la empresa.

\section{Enfoques de la calidad de servicio}

Los enfoques de la calidad de servicio se clasifican mediante las perspectivas del marketing y el management.

\section{Enfoque de márketing}

Este enfoque es desarrollado por la escuela nórdica y la escuela norteamericana. En cuanto a la primera escuela, Serrano, López, y García (2007), sostienen que la calidad es el resultado de la comparación entre el servicio esperado y recibido. Identifica dos dimensiones a la hora de hablar de calidad de servicio: calidad técnica y calidad funcional, siendo su interrelación un factor clave en la determinación de la imagen corporativa de la empresa en relación con la calidad. Enfoque conocido como modelo de la imagen, formulado por Grönroos, relaciona la calidad con la imagen corporativa percibida por los clientes como calidad técnica (qué se da) y la calidad funcional (cómo se da) asociada a los procesos (Dávila, Coronado y Cerecer, 2012). También, Clemenza, Gotera y Araujo (2010), agrega la dimensión imagen compuesta por rasgos proyectados hacia el exterior de la organización; las otras dos (técnica y funcional), son elementos internos de la organización.

En la segunda escuela, se concretan en tres aspectos básicos: a) la definición del constructo calidad de servicio; b) la creación de un instrumento de medida de la calidad de servicio, la escala SERVQUAL, con cinco dimensiones como constructo; y c) el desarrollo de un modelo de calidad de servicio basado en la existencia de gaps, que son procesos de comunicación y control para dirigir a los trabajadores que explican las diferencias entre el servicio esperado y recibido (Serrano, López, y García, 2007). Según, Duque (2005), la 
escuela Americana, denominada SERVQUAL, diseña el instrumento que aproxima la evaluación por separado de las expectativas y percepciones de un cliente mediante las dimensiones: a) tangibles, entendida como las facilidades físicas, equipamiento y apariencia del personal; b) confiabilidad, que es la habilidad de desempeñar el servicio prometido exactamente y con formalidad; c) sensibilidad, la cual definen como la buena voluntad para ayudar a los clientes y proveer un servicio oportuno; d) aseguramiento, que comprende el conocimiento y cortesía de los empleados y sus habilidades para inspirar confianza; e) empatía, que abarca la atención y cuidado individualizado que la compañía provee a sus clientes (Dávila, Coronado y Cerecer, 2012).

\section{Enfoque del management}

Sureshchandar, Rajendran y Anantharaman (2002) citado por Serrano, López, y García (2007), asumen que este enfoque examina la influencia de las dimensiones de la calidad de servicios en la percepción que los consumidores tienen de la misma, estos factores como variables soft del TQS (Total Quality Service, Calidad Total de los Servicios): gestión de los recursos humanos, enfoque a clientes, cultura de servicio, satisfacción de los empleados, compromiso y liderazgo de la alta dirección y responsabilidad social parecen ser más vitales que las dimensiones «hard» como factores influyentes sobre la percepción de la calidad de los servicios por parte de los consumidores. De ahí, Tarí (2000) menciona que actualmente todas las organizaciones son conscientes de su importancia y observamos cómo están reconociendo el papel estratégico tanto de la calidad del producto como de la dirección de la calidad o total quality management (TQM).

\section{Método}

El enfoque del trabajo se realizó bajo el enfoque cuantitativo porque mide las variables de estudio, aplicando el análisis estadístico. Es descriptivo correlacional simple, porque mediante el coeficiente de correlación se podrá concluir la relación que tiene o no el nivel de capacitación del capital humano con la calidad de servicio en los empresarios de las PYMES del centro comercial Plaza Josfel. Este tipo de estudio correlacional tiene como finalidad conocer la relación percibida que exista entre dos o más conceptos, categorías o variables en un contexto en particular (Hernández et al, 2010).

\section{Participantes}

La población del presente estudio está constituida por 1500 empresarios pertenecientes al centro comercial Plaza Josfel. Los participantes para este trabajo de investigación fueron determinados por el parámetro de muestra probabilística. Por lo tanto, el tamaño de la muestra estuvo conformado por 306 empresarios de las pequeñas empresas, los cuales fueron seleccionados a través del método estratificado proporcional a partir de lso rubros: ropa, descartables y plásticos, venta de mochilas, ferretería, abarrotes, celulares y soporte técnico, calzado, salón de belleza, grifería, piñatería, confección y sastrería, venta de frutas, juguería, restaurantes y comidas típicas, alquiler de ternos y vestidos, especerías y embutidos.

\section{Instrumentos}

El instrumento para medir la variable nivel del capital humano fue de elaboración propia a partir de los trabajos de Kaplan y Norton (2004), que propone la dimension de valor de activos de activos tangbles, el trabajo de Mungaray (2007) para la dimension de nivel de capacita- 
ción continua y nivel de años de experiencia. El cuestionario consta de 17 ítemes. Mediante la prueba piloto se obtuvo un confiabilidad 0.763 , lo cual refleja una consistencia interna aceptada.

Para medir la variable calidad se tomó como referencia la escala Servqual de Parasuraman, Zeithaml, y Berry (1994), utlizado por Carlos, Montelongo, Nájera (2010) de 18 preguntas que contiene las dimensiones: empatía, amabilidad, seguridad, capacidad de respuesta y solución de consulta. Ambos instrumentos valora las respuestas mediante la escala de Likert: $1=$ totalmente en desacuerdo, 2 = bastante en desacuerdo, $3=$ ni de acuerdo en desacuerdo, 4 = bastante de acuerdo y $5=$ totalmente de acuerdo.

\section{Resultados}

\section{Análisis descriptivo}

Tabla 1

Características sociodemográficas de los empresarios

Frecuencia Porcentaje

\begin{tabular}{llcc}
\hline \multirow{4}{*}{ Género } & Masculino & 90 & $29,4 \%$ \\
& Femenino & 216 & $70,6 \%$ \\
& Total & 306 & $100,0 \%$ \\
\cline { 2 - 3 } Edad & De 25 a 35 años & 68 & $22,2 \%$ \\
& De 36 a 46 años & 79 & $25,8 \%$ \\
& De 47 a 57 años & 111 & $36,3 \%$ \\
& De 58 años a más & 48 & $15,7 \%$ \\
& Total & 306 & $100,0 \%$ \\
\cline { 2 - 3 } & Primaria & 52 & $17,0 \%$ \\
& Secundaria & 104 & $34,0 \%$ \\
Nivel de & Completa & & \\
estudios & Secundaria incom- & 123 & $40,2 \%$ \\
& pleta & & \\
& Técnico & 17 & $5,6 \%$ \\
& Superior & 10 & $3,3 \%$ \\
& Total & 306 & $100,0 \%$ \\
\cline { 2 - 3 } Años de & De 0 a 5 años & 194 & $63,4 \%$ \\
trabajo en la & De 6 a 10 años & 98 & $32,0 \%$ \\
empresa & De 11 a 15 años & 14 & $4,6 \%$ \\
\hline & Total & 306 & $100,0 \%$ \\
\hline
\end{tabular}

Según la Tabla 1 se muestra las características sociodemográficas de los empresarios. El $29,4 \%$ es de género masculino, mientras que el 70,6\% es femenino. Asimismo, la mayoría (36,3\%) tiene entre los 47 a 57 años. Respecto al nivel de estudios, el 40,2\% tiene secundaria incompleta, mientras que el 3.3\% tiene estudios universitarios. La mayoría $(63,4 \%)$ tiene una antiguedad laboral, menos de 5 años en la empresa.

Tabla 2

Niveles de capacitación del capital humano y sus dimensiones

Frecuencia Porcentaje

\begin{tabular}{llcc}
$\begin{array}{l}\text { Nivel de capacitación del } \\
\text { capital humano }\end{array}$ & Bajo & 94 & $30,7 \%$ \\
& & & \\
& Medio & 124 & $40,5 \%$ \\
& Alto & 88 & $28,8 \%$ \\
Valor de los activos & Bajo & 194 & $63,4 \%$ \\
intangibles & & & \\
& Medio & 24 & $7,8 \%$ \\
& & & \\
& Alto & 88 & $28,8 \%$ \\
Nivel de capacitación & Bajo & 101 & $33,0 \%$ \\
continúa & & & \\
& Medio & 129 & $42,2 \%$ \\
& & & \\
& & & \\
& & & \\
Nivel de años de expe- & Bajo & 195 & $63,7 \%$ \\
riencia & & & \\
& Medio & 35 & $11,4 \%$ \\
& Alto & 76 & $24,8 \%$ \\
\hline
\end{tabular}

La Tabla 2 muestra los niveles de capacitación y sus dimensiones. El 40,5\% tiene un nivel medio de capacitación. En cuanto al valor de los activos intangibles, la capacitación está en un nivel bajo $(63,4 \%)$. El 42,2\% se encuentra en un nivel medio de capacitación continua. El $63,4 \%$ se encuentra en un nivel bajo de capacitación respecto a los años de experiencia. 
Tabla 3

Niveles de calidad de servicio y sus dimensiones

\begin{tabular}{|c|c|c|c|}
\hline & & Frecuencia & Porcentaje \\
\hline \multirow[t]{4}{*}{ Calidad de servicio } & Bajo & 174 & $56,9 \%$ \\
\hline & Medio & 51 & $16,7 \%$ \\
\hline & Alto & 81 & $26,5 \%$ \\
\hline & Total & 306 & $100,0 \%$ \\
\hline \multirow{4}{*}{$\begin{array}{l}\text { Atención a tiempo de } \\
\text { pedido }\end{array}$} & Bajo & 93 & $30,4 \%$ \\
\hline & Medio & 129 & $42,2 \%$ \\
\hline & Alto & 84 & $27,5 \%$ \\
\hline & Total & 306 & $100,0 \%$ \\
\hline \multirow[t]{4}{*}{ Buen trato al cliente } & Bajo & 190 & $62,1 \%$ \\
\hline & Medio & 25 & $8,2 \%$ \\
\hline & Alto & 91 & $29,7 \%$ \\
\hline & Total & 306 & $100,0 \%$ \\
\hline \multirow{4}{*}{$\begin{array}{l}\text { Entrega oportuna de pe- } \\
\text { dido }\end{array}$} & Bajo & 66 & $21,6 \%$ \\
\hline & Medio & 159 & $52,0 \%$ \\
\hline & Alto & 81 & $26,5 \%$ \\
\hline & Total & 306 & $100,0 \%$ \\
\hline \multirow[t]{4}{*}{ Calidad de servicio } & Bajo & 174 & $56,9 \%$ \\
\hline & Medio & 51 & $16,7 \%$ \\
\hline & Alto & 81 & $26,5 \%$ \\
\hline & Total & 306 & $100,0 \%$ \\
\hline
\end{tabular}

La Tabla 3 presenta los niveles de calidad de servicio y sus dimensiones. El $56,9 \%$ percibe que ofrece un nievel bajo de calidad. En cuanto a la atención a tiempo de pedido, la la calidad de servicio está en un nivel medio (42,2\%); el buen trato al cliente, en un nivel bajo $(62,1 \%)$; la entrega oportuna de pedido, en un nivel medio (52.0\%).

\section{Prueba de hipótesis}

Hipótesis general

- Hipótesis nula $\left(\mathrm{H}_{0}\right)$ : El nivel de capacitación del capital humano no se relaciona favorablemente con la calidad de servicio en los empresarios de las PYMES del centro comercial Plaza Josfel, en la localidad de Ate Vitarte, en el período 2016.
- Hipótesis alterna $\left(\mathrm{H}_{1}\right)$ : El nivel de capacitación del capital humano se relaciona favorablemente con la calidad de servicio en los empresarios de las PYMES del centro comercial Plaza Josfel, en la localidad de Ate Vitarte, en el período 2016.

\section{Regla de decisión}

Si p valor $>0.05$, se acepta la hipótesis nula $\left(\mathrm{H}_{0}\right)$

Si $p$ valor $<0.05$, se rechaza la hipótesis nula $\left(H_{0}\right)$, por lo tanto se acepta la hipótesis alterna

Tabla 4

Prueba de hipótesis para la relación entre nivel de capacitación del capital humano y calidad de servicio

Calidad de servicio

Chi-cuadrado gl pvalor

Nivel de capacitación del $169.602 \quad 4 \quad, 000^{\star}$

capital humano

*. El estadístico de chi-cuadrado es significativo en el nivel ,05

La Tabla 4 presenta la relación significativa entre el nivel de capacitación del capital humano y calidad de servicio, obteniéndose un Chi cuadrado $=169.602$ con un $p$ valor 0.000 , indicando que se rechaza la hipótesis alterna. Es decir, que a mayor el nivel de capacitación del capital humano mejora la calidad de servicio en los empresarios de las PYMES del centro comercial Plaza Josfel, en la localidad de Ate Vitarte, en el período 2016.

\section{Hipótesis específica 1}

Para comprobar las hipótesis específicas se procedió a relacionar nivel de capacitación del capital humano con cada una de las dimensiones de calidad de servicio. Se obtuvieron los siguientes resultados. 
- Hipótesis nula $\left(\mathrm{H}_{0}\right)$ : El nivel de capacitación del capital humano no se relaciona favorablemente con la atención a tiempo de pedido en los empresarios de las PYMES del centro comercial Plaza Josfel, en la localidad de Ate Vitarte, en el período 2016.

- Hipótesis alterna $\left(\mathrm{H}_{1}\right)$ : El nivel de capacitación del capital humano se relaciona favorablemente con la atención a tiempo de pedido en los empresarios de las PYMES del centro comercial Plaza Josfel, en la localidad de Ate Vitarte, en el período 2016.

\section{Regla de decisión}

Si p valor $>0.05$, se acepta la hipótesis nula $\left(\mathrm{H}_{0}\right)$

Si $p$ valor $<0.05$, se rechaza la hipótesis nula $\left(H_{0}\right)$, por lo tanto se acepta la hipótesis alterna

Tabla 5

Prueba de hipótesis para la relación entre el nivel de capacitación del capital humano y la atención a tiempo de pedido

\section{Calidad de servicio}

\begin{tabular}{lccc} 
& Chi-cuadrado & gl & pvalor \\
$\begin{array}{l}\text { Atención a tiempo de pe- } \\
\text { dido }\end{array}$ & & & \\
\hline
\end{tabular}

*. El estadístico de chi-cuadrado es significativo en el nivel,05.

La Tabla 5 presenta la relación significativa entre el nivel de capacitación del capital humano y la atención a tiempo de pedido, obteniéndose un Chi cuadra$d o=287.967$ con un $p$ valor 0.000 , indicando que se rechaza la hipótesis alterna. Es decir, que a mayor el nivel de capacitación del capital humano mejora la atención en los empresarios de las PYMES del centro comercial Plaza Josfel, en la localidad de Ate Vitarte, en el período 2016.

\section{Hipótesis específica 2}

- Hipótesis nula $\left(\mathrm{H}_{0}\right)$ : El nivel de capacitación del capital humano no se relaciona favorablemente con buen trato al cliente en los empresarios de las PYMES del centro comercial Plaza Josfel, en la localidad de Ate Vitarte, en el período 2016.

- Hipótesis alterna $\left(\mathrm{H}_{1}\right)$ : El nivel de capacitación del capital humano se relaciona favorablemente con buen trato al cliente en los empresarios de las PYMES del centro comercial Plaza Josfel, en la localidad de Ate Vitarte, en el período 2016.

\section{Regla de decisión}

Si p valor $>0.05$, se acepta la hipótesis nula $\left(\mathrm{H}_{0}\right)$

Si p valor $<0.05$, se rechaza la hipótesis nula $\left(H_{0}\right)$, por lo tanto se acepta la hipótesis alterna

Tabla 6

Prueba de hipótesis para medir la relación entre el nivel de capacitación del capital humano y buen trato al cliente

Calidad de servicio

$$
\text { Chi-cuadrado Gl pvalor }
$$

Buen trato al cliente $\quad 134.655 \quad 4 \quad, 000^{*}$

*. El estadístico de chi-cuadrado es significativo en el nivel ,05.

La Tabla 6 presenta la relación significativa entre el nivel de capacitación del capital humano y la buen trato al cliente, obteniéndose un Chi cuadrado=134.655 con un p valor 0.000 , indicando que se rechaza la hipótesis nula. Es decir, que a mayor el nivel de capacitación del capital humano mejora el buen trato a los clientes en los empresarios de las PYMES del centro comercial Plaza Josfel, en la localidad de Ate Vitarte, en el período 2016. 
Hipótesis específica 3

- Hipótesis nula $\left(\mathrm{H}_{0}\right)$ : El nivel de capacitación del capital humano no se relaciona favorablemente con la entrega oprotuna de pedido en los empresarios de las PYMES del centro comercial Plaza Josfel, en la localidad de Ate Vitarte, en el período 2016.

- Hipótesis alterna $\left(\mathrm{H}_{1}\right)$ : El nivel de capacitación del capital humano se relaciona favorablemente con la entrega oportuna de pedido en los empresarios de las PYMES del centro comercial Plaza Josfel, en la localidad de Ate Vitarte, en el período 2016.

\section{Regla de decisión}

Si $p$ valor $>0.05$, se acepta la hipótesis nula $\left(\mathrm{H}_{0}\right)$

Si $\mathrm{p}$ valor $<0.05$, se rechaza la hipótesis nula $\left(H_{0}\right)$, por lo tanto se acepta la hipótesis alterna

Tabla 7

Prueba de hipótesis para la relación entre el nivel de capacitación del capital humano y la entrega oportuna de pedido

Calidad de servicio

Chi-cuadrado Gl pvalor

$\begin{array}{llll}\text { Entrega oportuna de pedido } & 238.980 \quad 4 & 4 & , 000^{*}\end{array}$

*. El estadístico de chi-cuadrado es significativo en el nivel ,05.

La Tabla 7 presenta la relación significativa entre el nivel de capacitación del capital humano y la entrega oportuna de pedido, obteniéndose un Chi cuadrado $=238.980$ con un $p$ valor 0.000 , indicando que se rechaza la hipótesis nula. Es decir, que a mayor el nivel de capacitación del capital humano mejora la entrega oportuna de pedido en los empre- sarios de las PYMES del centro comercial Plaza Josfel, en la localidad de Ate Vitarte, en el período 2016.

\section{Discusión}

El trabajo de investigación ha permitido establecer el nivel de capacitación y la calidad del servicio que brindan los empresarios de las PYMES del centro comercial Plaza Josfel, siendo la capacitación un factor muy relevante en la productividad relacionada a la satisfacción del cliente como indicador de la noción actual de calidad.

Según el coeficiente (169.602) que establece una correlación positiva significativa ( $p$ valor 0.000 ), se toma la decisión de rechazar la hipótesis nula y se acepta la hipótesis alterna, concluyendo que existe relación directa y significativa entre el nivel de capacitación del capital humano y la calidad de servicio en los empresarios de las PYMES del centro comercial Plaza Josfel. Según Najul Godoy (2011), la importancia de la calidad radica en la actualización de los procesos e integrar nuevas tecnologías a los mismos; es decir, la aplicación de la calidad y la transmisión de los conocimientos específicos relativos al trabajo incidicen en la productividad de la empresa.

Según Shultz (1960), citado por De la Rica y Iza (2013), la teoría del capital humano relaciona de una manera directa la inversión de capital humano con un aumento de la productividad individual derivado de un aumento en los conocimientos del individuo. En ese sentido, los niveles de capacitación del capital humano y sus dimensiones se encuentran en un nivel medio (40.5\%), entendiéndose que se necesita mayor oportunidad de capacitación.

Según (Najul Godoy, 2011), la calidad y la transmisión de los conocimientos es- 
pecíficos relativos al trabajo están relacionados con la productividad de la empresa, en consecuencia la actualización de competencias permite perfeccionar o corregir las transformaciones y cuyos resultados conllevan al crecimiento de las empresas.

En el estudio se observa respecto al nivel de estudios, el 17,0\% tiene un nivel primario, el $34,0 \%$ tiene secundaria completa, el $40,2 \%$ con secundaria incompleta, mientras que en el $5,6 \%$ tiene nivel de estudio técnico y, solamente, un $3,3 \%$ tiene un nivel de estudio superior. En ese sentido, Arrow (1973), citado por De la Rica y lza (2013), menciona que la educación, y más concretamente la educación superior, actúa como un filtro que clasifica a los individuos, pero sin aportarles necesariamente ninguna característica que aumente su productividad. Asimismo Mungaray y Ramírez (2007), en sus estudio concluye que las personas con mayores niveles de escolaridad alcanzada posee mayor experiencia en cuanto al manejo de los negocios.

\section{Conclusiones}

Respecto al objetivo general se concluye que existe una relación significativa entre el nivel de capacitación del capital humano y la calidad de servicio en los empresarios de las PYMES del centro comercial Plaza Josfel, en el periodo 2016.

Respecto al primer objetivo específi$\mathrm{co}$, se concluye que existe una relación significativa entre el nivel de capacitación del capital humano con la atención a tiempo de pedido en los empresarios de las PYMES del centro comercial Plaza Josfel, en el período 2016.

En cuanto al segundo objetivo específico, se concluye que existe una relación significativa entre el nivel de capacitación del capital humano y el buen trato al cliente en los empresarios de las PYMES del centro comercial Plaza Josfel, periodo 2016.

Respecto al tercer objetivo específico, se concluye que existe una relación significativa entre el nivel de capacitación del capital humano con la entrega oportuna del pedido en los empresarios de las PYMES del centro comercial Plaza Josfel, 


\section{Referencias}

Alvarado Daga, G. E. (2013). La inversión en la capacidad del capital humano y la metodología de la gestión por competencias. Quipukamayoc Revista de la Facultad de Ciencias Contables, 21(40), 81-93.

Becerra, F., y Álvarez, G. C. (2011). El talento humano y la innovación empresarial en el contexto de las redes empresariales: el clúster de prendas de vestir en Caldas Colombia. Tesis de maestría, Universidad Nacional de Colombia.

Calderón Hernández, G., y Naranjo Valencia, J. C. (2004). Competencias laborales de los gerentes de talento humano. Innovar, 14(23), 79-97.

Carlos, O. C., Montelongo, C. Y., y Nájera, G. M. (2010). La calidad del servicio de un centro de información. Conciencia Tecnológica. (40), 5-9.

Clemenza, C., Gotera, A., y Araujo, R. (2010). Calidad de los servicios prestados por el Servicio Autónomo Municipal de Administración Tributaria. Revista Venezolana de Gerencia, 15(49), 103-124.

Cotte Poveda, A., y Cotrino Sossa, J. A. (2006). Crecimiento económico y distribución del ingreso en Colombia: evidencia sobre el capital humano y el nivel de educación. Cuadernos de Administración, 19(32), 337-356.

Dávila, B. M., Coronado, Q. J., y Cerecer, C. B. (2012). Las dimensiones de la calidad del servicio en el proceso de distribución y comercialización de energía eléctrica. Contaduría y Administración, 57(3), 175-195.
De la Rica Goiricelaya, S., y Padilla, A. I. (1999). Capital humano, productividad y crecimiento: teorías y contrastes. Ekonomiaz: Revista Vasca de Economía, (45), 266-283.

Destinobles, A. G. (2006). El capital humano en las teorías del crecimiento económico. Textos Universitarios. Universidad Autónoma de Chihuahua.

Didier Pino, N. (2013). A través de las nociones de capital humano. Revista Iberoamericana de Psicología Ciencia y Tecnología, 6(2), 7-16.

Duque, O. E. (2005). Del servicio y sus modelos de medición. Innovar Revista de Ciencias Administrativas y Sociales, 15(25), 64-80.

García Pérez, E. (1992). La estimacion de la tasa de rendimiento de la inversión en educación: una aplicación al caso de la enseñanza media en España. Tesis de maestría, Universidad Complutense de Madrid. Facultad de Ciencias Económicas y Empresariales.

García Santillán, A., y Edel Navarro, R. (2008). El capital humano en las organizaciones. México: Universidad Veracruzana.

Kaplan, R., Y Norton, D. (2004). Medir la disposición estratégica de los activos intangibles. Harvard Business Review, 84(2), 3-13.

Lara, L. R. (2002). La gestión de la calidad en los servicios. Instituto Tecnológico de Aguascalientes, Conciencia Tecnológica, (19).

Lozano Correa, L. J. (2007). El talento humano, una estrategia de éxito en las empresas culturales. Revista Escuela 
de Administración de Negocios, (60), 147-164.

Madrigal Torres, B. E. (2009). Capital humano e intelectual: su evaluación. Observatorio Laboral Revista Venezolana, 2(3), 65-81.

ManpowerGroup. (2013). Encuesta de escasez de talento 2013. Investigaciones: ManpowerGroup, 1-25.

Mungaray, A., y Ramírez Urquidy, M. (2007). Capital humano y productividad en microempresas. México: Universidad Autonoma de Baja California.

Najul Godoy, J. (2011). El capital humano en la atención al cliente y la calidad de servicio. Observatorio Laboral Revista Venezolana, 4(8), 23-35.

Ospina, G. W., Stella, R. L., y Estrada, M. S. (2004). Capital humano y conocimiento. Retos para la empresa del siglo XXI. Scientia Et Technica, 2(25), 215-219.

Parasuraman, A., Zeithaml, V. A., y Berry, L. L. (1994). Alternative scales for measuring service quality: a comparative assessment based on psychometric and diagnostic criteria. Journal of retailing, 70(3), 201-230.

Pinto, M., Fernández, V., y Gómez, C. (2007). La herramienta BiQual como instrumento para el estudio de la calidad del servicio en bibliotecas universitarias españolas de ciencia y tecnología. Revista Española de Documentación Científica, 30(4), 465491.

Rivera Sánchez, F. (2005). Capacitación y formación permanente. Un diagnóstico del proyecto redes educativo-productiva de Chilecalifica, en las pers- pectivas de los desafios a enfrentar en el tránsito hacia la sociedad del conocimiento. Tesis de maestría, Santiago: Universidad de Chile.

Santos Jiménez, N. (2012). El capital humano en empresas peruanas de generación eléctrica. Industrial Data, 15(1), 063-069.

Selva, S. C. (2004). El capital humano y su contribucion al crecimiento económico. España: Ediciones de la Universidad de Castilla. La Mancha. Ed. II. Título III. Serie, 331(2), 460-28.

Serrano, B. A., López, F. C., y García, P. G. (2007). Gestión de la calidad en servicios: una revisión desde la perspectiva del management. Cuadernos de Gestión, 7(1), 33-49.

Tarí Guilló, G. J. (2000). Calidad total: fuente de ventaja competitiva. Murcia: Publicaciones Universidad de Alicante.

Torres, M. E., y Araya, C. L. (2010). Construcción de una escala para medir la calidad del servicio de las universidades: una aplicación al contexto chileno. Revista de Ciencias Sociales, 16(1).

Valencia Rodríguez, M. (2005). El capital humano, otro activo de su empresa. Entramado, 1(2), 20-33.

Villalobos, M. G., y Pedroza, F. R. (2009). Perspectiva de la teoría del capital humano acerca de la relación entre educación y desarrollo económico. Tiempo de Educar, 10(20), 273-306.

Werther, W., Davis, K., y Guzmán, B. M. (2014). Administración de recursos humanos. Gestión del capital humano. México: McGraw-Hill Interamericana. 
